\title{
Performance of an optical readout GEM-based TPC
}

\author{
L.M.S. Margato ${ }^{a, *}$,F.A.F. Fraga ${ }^{a, b}$, S.T.G. Fetal ${ }^{a}$, M.M.F.R. Fraga ${ }^{a}$, \\ E.F.S. Balau ${ }^{\mathrm{a}, \mathrm{b}}$, A. Blanco ${ }^{\mathrm{a}}$, R. Ferreira Marques ${ }^{\mathrm{a}, \mathrm{b}}$, A.J.P.L Policarpo ${ }^{\mathrm{a}, \mathrm{b}}$ \\ ${ }^{a}$ LIP_Coimbra and Departamento de Física da Universidade de Coimbra, 3004-516 Coimbra, Portugal \\ ${ }^{\mathrm{b}}$ CFRMUC-Centro de Física da Radiação e de Materiais da Universidade de Coimbra, \\ Departamento de Física da Universidade de Coimbra, 3004-516 Coimbra, Portugal
}

Available online 12 August 2004

\begin{abstract}
We report on the operation of a GEM-based small TPC using an optical readout. The detector was operated with a mixture of $\mathrm{Ar}+\mathrm{CF}_{4}$ using $5.48 \mathrm{MeV}$ alpha particles obtained from a ${ }^{241} \mathrm{Am}$ source and the GEM scintillation was concurrently read by a CCD camera and a photomultiplier. Precision collimators were used to define the track orientation. Qualitative results on the accuracy of the track angle, length and charge deposition measurements are presented.
\end{abstract}

(C) 2004 Elsevier B.V. All rights reserved.

PACS: 29.40.Cs; 29.40.Gx; 29.40.Mc

Keywords: GEM detectors; CCD; Scintillation; Optical readout; Time projection chamber; Neutron spectrometry

\section{Introduction}

It has been shown that when operated with adequate gas mixtures, the gas electron multiplier (GEM [1]) scintillation can be used for track imaging using integrating devices (CCDs) and photomultipliers $[2,3]$. In the last work, it was also shown that the light signals taken from a photomultiplier were faster than the charge signals and that their rise time was correlated with the track

\footnotetext{
*Corresponding author. Tel.: + 351-39-833-465; fax: + 35139-822-358.

E-mail address: margato@lipc.fis.uc.pt (L.M.S. Margato).
}

orientation, suggesting its use for the determination of the track angle in TPCs.

The aim of this work is to demonstrate that complete information of track details can be extracted from the photomultiplier signal and thus a new generation of instruments can be designed based on this concept.

\section{The experimental set-up}

Using a small-volume TPC $(\sim 0.51)$, similar to the one described in Ref. [4], coupled with a 
readout using a CCD camera (Hamamatsu C4880-21) with triggering capability, a photomultiplier and a fast $4 \mathrm{G}$ sample/s digitising oscilloscope (Tektronix TDS7104), which made simultaneous recording of the track projection image and the light and charge pulses possible, we collected and analysed data on this mode of operation. The experiments were performed with a ${ }^{241} \mathrm{Am}$ alpha source and several precision collimators were used for data taking, defining tracks of $15,30,45,60$ and $90^{\circ}$.

The gas mixture was $\mathrm{Ar}+5 \% \mathrm{CF}_{4}$ and the track length was approximately $4 \mathrm{~cm}$. The detector was operated in a continuous flux system mode and the gases used, all of high-purity research grade, were supplied to the chamber through mass flowmeters without further purification. The pressure inside the chamber was monitored using a pressure gauge and the track lengths were corrected for the

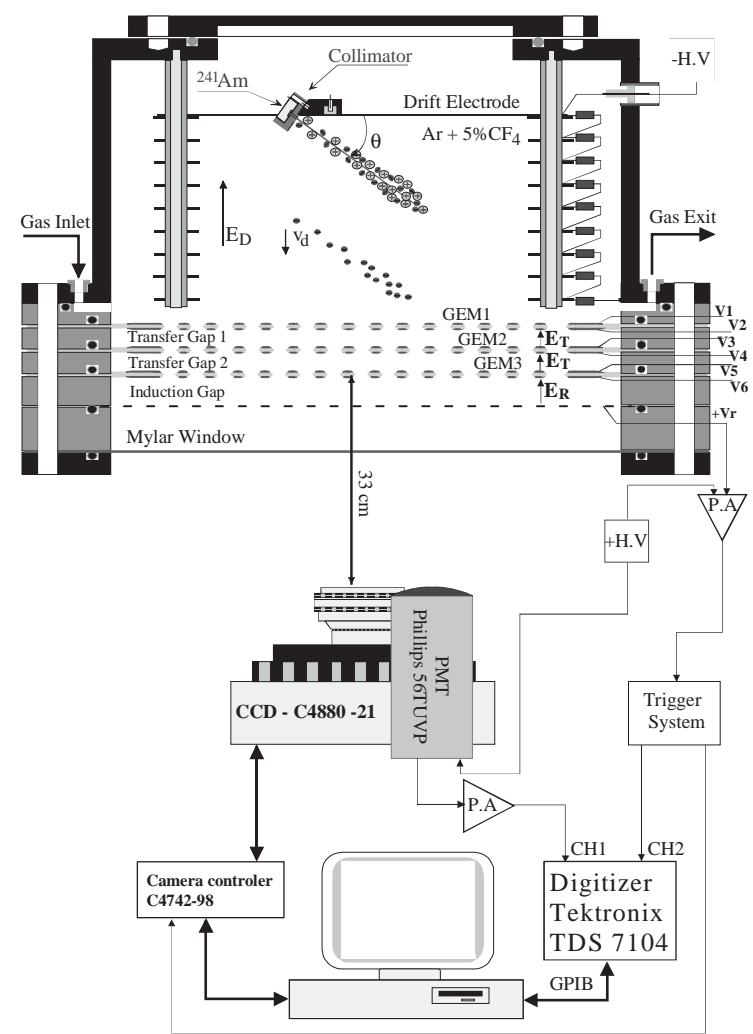

Fig. 1. Schematic diagram of the experimental setup. The scintillation of the triple GEM camera was read out by a CCD and a photomultiplier. variation of the pressure during the experimental measurements.

The schematic drawing of the detector system used in this work is shown in Fig. 1. The triple GEM-based TPC is able to image track lengths up to $8 \mathrm{~cm}$ and is fitted with a Mylar window for the output of the scintillation light.

A drift grid, placed before the first GEM, defines the absorption zone. The GEMs were supported by glass fibre frames, and the distance between the GEMs was $2 \mathrm{~mm}$. An optically transparent collecting grid was placed $3 \mathrm{~mm}$ away from the final GEM, thereby defining the induction gap. The $10 \times 10 \mathrm{~cm}^{2}$ GEMs had $80 \mu \mathrm{m}$ metal diameter holes and $70 \mu \mathrm{m}$ bi-conical kapton holes. The kapton thickness was $50 \mu \mathrm{m}$ and the pitch was $140 \mu \mathrm{m}$ for all GEMs. The CCD and the photomultiplier (Philips 56TUVP-type B-extended S20) were placed approximately $33 \mathrm{~cm}$ away from the third GEM plane for the readout of the scintillation light.

Drift field $(0.5 \mathrm{kV} / \mathrm{cm})$ and GEMs voltage, $V_{\mathrm{GEM}}=380 \mathrm{~V}$ (voltage across each GEM), were set to optimise the electron drift velocity [5] and the charge transfer (transfer field $E_{\mathrm{T}}=1.3 \mathrm{kV} / \mathrm{cm}$ ) [7], respectively.

The induction electrode signals were used to post-trigger both the CCD shutter and the scope, simultaneously acquiring the individual track image and the respective charge and photomultiplier signals.

\section{Experimental results}

The track orientation was defined by accurate collimators of $15,30,45,60$ and $90^{\circ}$, and a set of 100 alpha track images were acquired on coincidence with the photomultiplier waveforms for each angle. Image and waveform processing were performed and track projection length and energy and rise time of photomultiplier signals were computed. For each set of measurements, CCD and photomultiplier signals energy resolution were estimated as also the photomultiplier signals time resolution.

The analysis software was developed using the Matlab image and signal processing tools. The 
CCD images were corrected for background pixel noise, and the tracks were rotated in order to study the projection of the Bragg curve of alpha tracks on a GEM plane, that supplied the information about the energy and track length. Fig. 2 shows an example of track image processing. The vertical and horizontal track profiles of the track charge density are also shown.
The orientation of the track can be extracted from the rise time $(\tau)$ of the photomultiplier pulse (Fig. 3). Considering that $\mathbf{R}_{\mathbf{z}}$ is the distance measured along the drift-field direction between the beginning and end of the track, and $\mathbf{v}_{\mathbf{d}}$ is the electron drift velocity of $\mathrm{Ar}+5 \% \quad \mathrm{CF}_{4}$ [5], $\mathbf{R}_{\mathbf{z}}=\tau \cdot \mathbf{v}_{\mathbf{d}}$. Therefore, $\boldsymbol{\theta}=$ arcsine $\left(\mathbf{R}_{\mathbf{z}} / \mathbf{R}\right)$, $\mathbf{R}$ being the range of the alpha particle computed using the

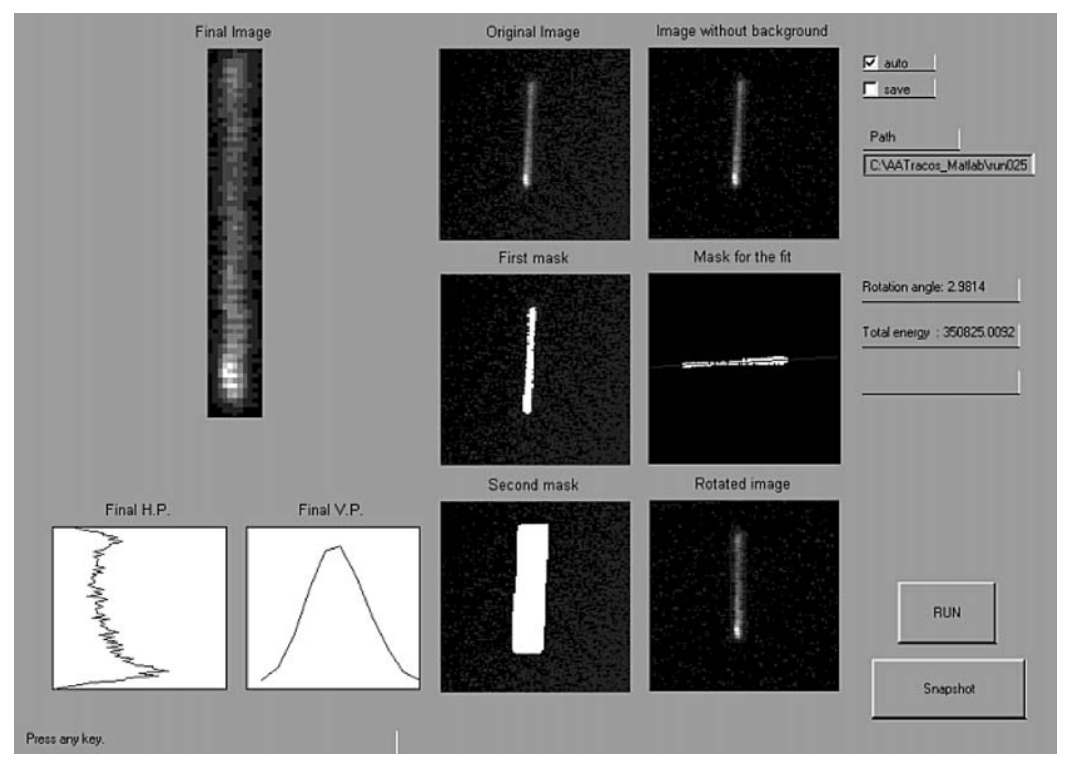

Fig. 2. Image processing of one-track CCD image.

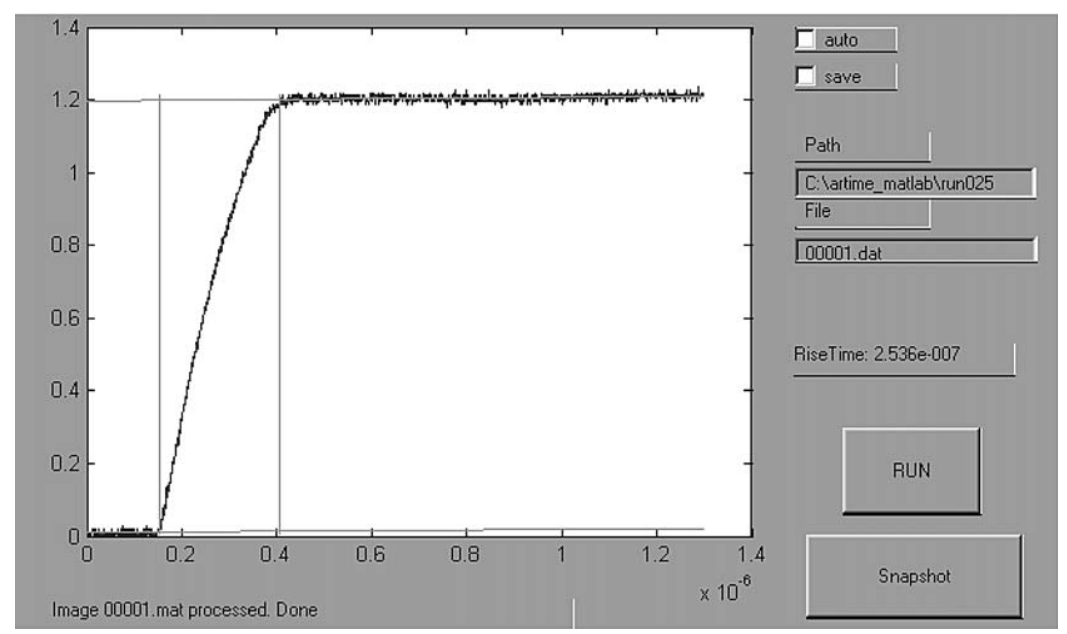

Fig. 3. Typical signal shape of the PMT preamplifier. 
SRIM code [6]. The same information can also be extracted from the length of the track projection on the GEM plane, $\mathbf{R}_{\mathbf{p}}$, as the track angle $\boldsymbol{\theta}$ equates arccosine $\left(\mathbf{R}_{\mathbf{p}} / \mathbf{R}\right)$.

A set of 100 photomultiplier waveforms and respective track images have been acquired for each collimator angle, statistical analysis was carried out for every set of data. The computed angle data, for each angle, was fitted with a Gaussian distribution. The width is due to dispersible effects such as track-range straggling, electron diffusion, and the collimator aperture, which is the dominant effect in this experiment.

As expected, the mean angle track computed from the risetime depends linearly on the angle defined by the collimators, as shown in Fig. 4.

The track angular spread due to the collimator characteristics was $2^{\circ}$ (rms), as obtained by simulation and later confirmed evaluating the CCD image of the beam profile at $90^{\circ}$. The width of the measured angular distribution is shown in greater detail in Fig. 5.

If the beam width is deconvoluted, the angle track can be determined from the photomultiplier data with a typical standard deviation smaller than $2^{\circ}$ for an angle less than $60^{\circ}$. For larger angles the longitudinal straggling, around $1.6 \mathrm{~mm}$, should be considered.

As we considered the development of a gaseous recoil neutron spectrometer for fast neutrons [8]

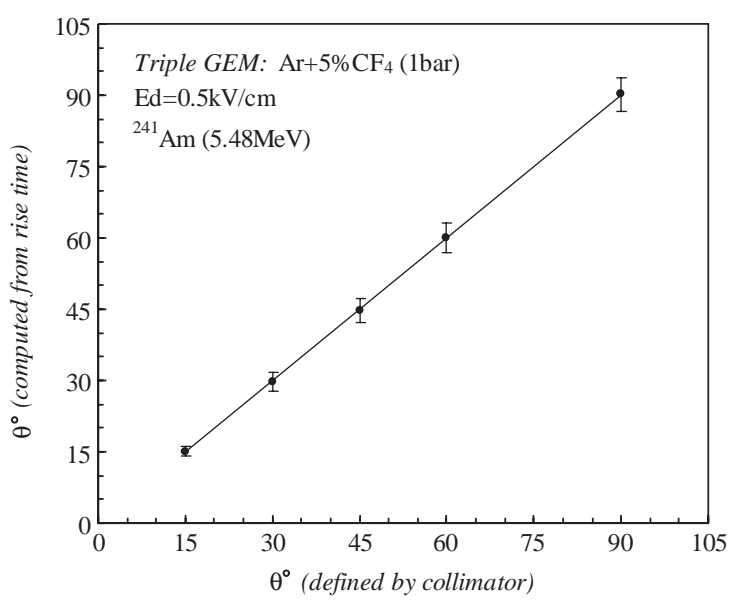

Fig. 4. Track angle orientation computed from light signal rise time versus track angle defined by collimator.

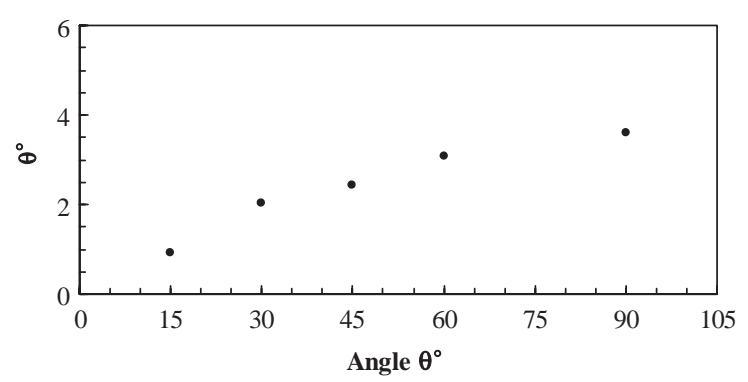

Fig. 5. Standard deviation of angle computed from rise time versus track angle.

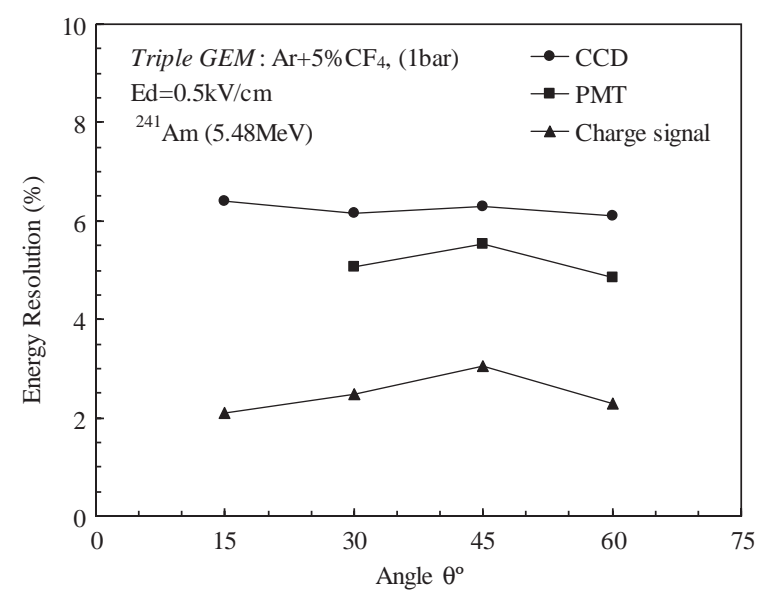

Fig. 6. Energy resolution measured from the CCD images and from the charge and photomultiplier signals versus track angle.

based on this technique, the determination of the energy of the track is primordial [9]. The energy resolution of the track energy measurements using the CCD, photomultiplier and induced charges is presented in Fig. 6.

The energy resolution obtained using the photomultiplier was limited by photon statistics as the distance between the photomultiplier and the detector is too large $(\sim 30 \mathrm{~cm})$, to avoid shadowing the CCD in this setup. Using an improved position an energy resolution similar to that obtained with charge signals could be measured (see Fig. 3 of Ref. [3]). Since the number of scintillating photons produced per track and imaged by the CCD is sufficiently high to neglect the CCD electronic and dark current noise, the CCD energy resolution is similar to the energy resolution presented by photomultiplier signals. 


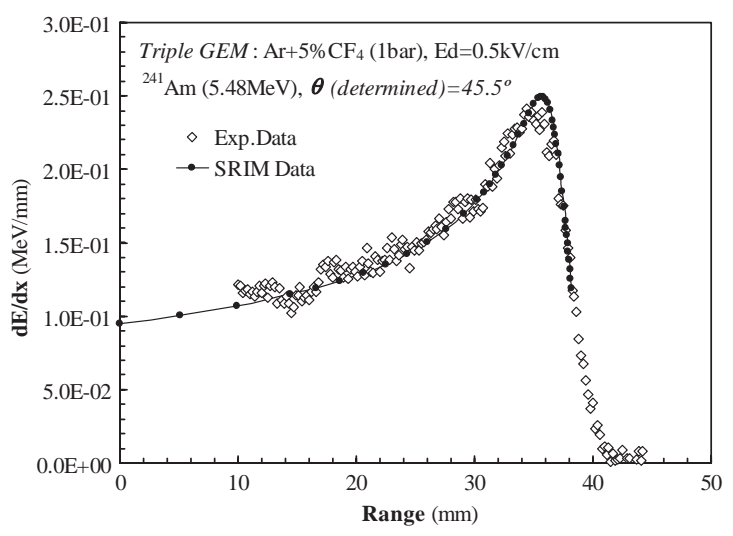

Fig. 7. Determination of the track orientation by a fit of the Bragg curve imaged by the CCD using the SRIM simulation.

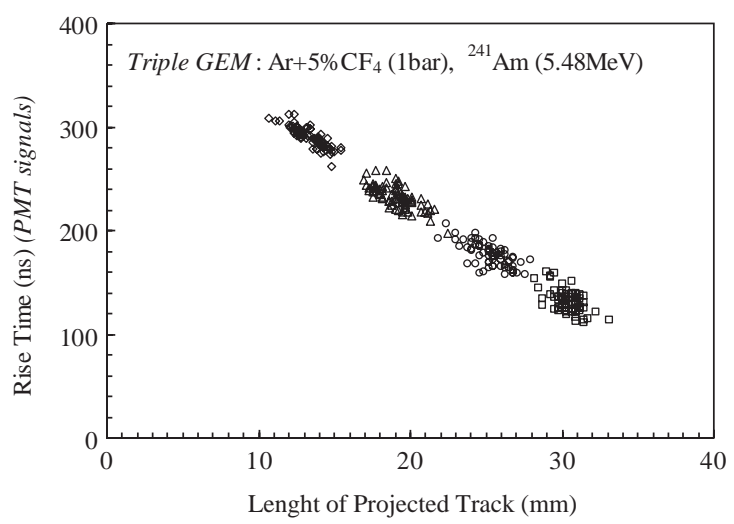

Fig. 8. Correlation between light signal rise time and length of the projected track.

As referred the angle track could be determined from the energy and the Bragg curve of a projection track. Fig. 7 shows the best fit of the projection of a single track to the Bragg curve simulated with the SRIM code, using a $45^{\circ}$ collimator, showing very good accuracy.

The results presented in Fig. 8 confirm an excellent correlation between the rise time and the length of projection track suggesting that a better angle resolution can be achieved.

\section{Conclusions}

It was shown that the track angle can be determined with an accuracy better than $2^{\circ}$ using the photomultiplier signal rise time information, that combined with the CCD image of the projection gives full track particulars, including the density of the charge deposit. It has been demonstrated that the track angle can also be determined from the Bragg curve data. Moreover, if a current preamplifier is used instead of a charge preamplifier, the pulse shape reflects the energy density along the track, an important highlight considering that the $\mathrm{d} E / \mathrm{d} x$ information can be used to identify different types of particles and is suitable for discrimination purposes, for example, the rejection of the gamma background in thermal neutron detectors or background rejection in dark matter experiments.

The light readout is of great interest either for general TPC readout or other types of detectors relying on the determination of track orientation, and is currently being applied to the ongoing development of a neutron recoil spectrometer based on GEM scintillation and an X-ray polarimeter.

\section{Acknowledgements}

L.M.S. Margato was supported by the FCT (Fundação para a Ciência e Tecnologia) SFRH/ $\mathrm{BD} / 6786 / 2001$ research grant.

\section{References}

[1] F. Sauli, Nucl. Instr. and Meth. A 368 (1997) 531.

[2] F.A.F. Fraga, et al., Nucl. Instr. and Meth. A 513 (2003) 379.

[3] L.M.S. Margato, et al., Nucl. Instr. and Meth. A 504 (2003) 374.

[4] F.A.F. Fraga, et al., IEEE Trans. Nucl. Sci. 49 (1) (2002) 281.

[5] M. Kurihara, et al., J. Phys. D: Appl. Phys 33 (2000) 2146.

[6] J.F. Ziegler, J.P. Biersack, SRIM2003, copyright: SRIM.com, http://www.srim.org.

[7] S. Roth, et al., Nucl. Instr. and Meth. A 513 (2003) 379.

[8] G. Wakabayashi, et al., IEEE Trans. Nucl. Sci. 48 (1) (2001) 320.

[9] G.F. Knoll, Radiation Detection and Measurement, third ed., Wiley, New York, 2000. 